\title{
A Fast Projection Method for Connectivity Constraints in Image Segmentation
}

\author{
Jan Stühmer and Daniel Cremers \\ Department of Computer Science, Technische Universität München, Germany
}

\begin{abstract}
We propose to solve an image segmentation problem with connectivity constraints via projection onto the constraint set. The constraints form a convex set and the convex image segmentation problem with a total variation regularizer can be solved to global optimality in a primal-dual framework. Efficiency is achieved by directly computing the update of the primal variable via a projection onto the constraint set, which results in a special quadratic programming problem similar to the problems studied as isotonic regression methods in statistics, which can be solved with $O(n \log n)$ complexity. We show that especially for segmentation problems with long range connections this method is by orders of magnitudes more efficient, both in iteration number and runtime, than solving the dual of the constrained optimization problem. Experiments validate the usefulness of connectivity constraints for segmenting thin structures such as veins and arteries in medical image analysis.
\end{abstract}

\section{Introduction}

To allow to preserve thin structures, topological constraints, and especially those that preserve connectivity $[16,15]$, have been introduced into image segmentation methods.

These constraints have a great advantage in several application areas, including the segmentation of arteries and veins in medical imaging but also in a user interactive setting for general image segmentation. They are very useful when thin structures should be extracted from image data, allowing to extract the whole branching tree of blood vessels in the lung, as shown on the left in Fig. 1. For comparison, a total variation regularized segmentation of the dataset without connectivity constraints is shown on the right. In order to preserve the thin structures, only a very small weight of the regularizer can be chosen. Therefore a lot of noise is still present in the final segmentation.

Including these constraints in the segmentation model either leads to a higher algorithmic complexity $[16,6]$ or slow convergence when solving the dual of the constrained optimization problem [15]. 

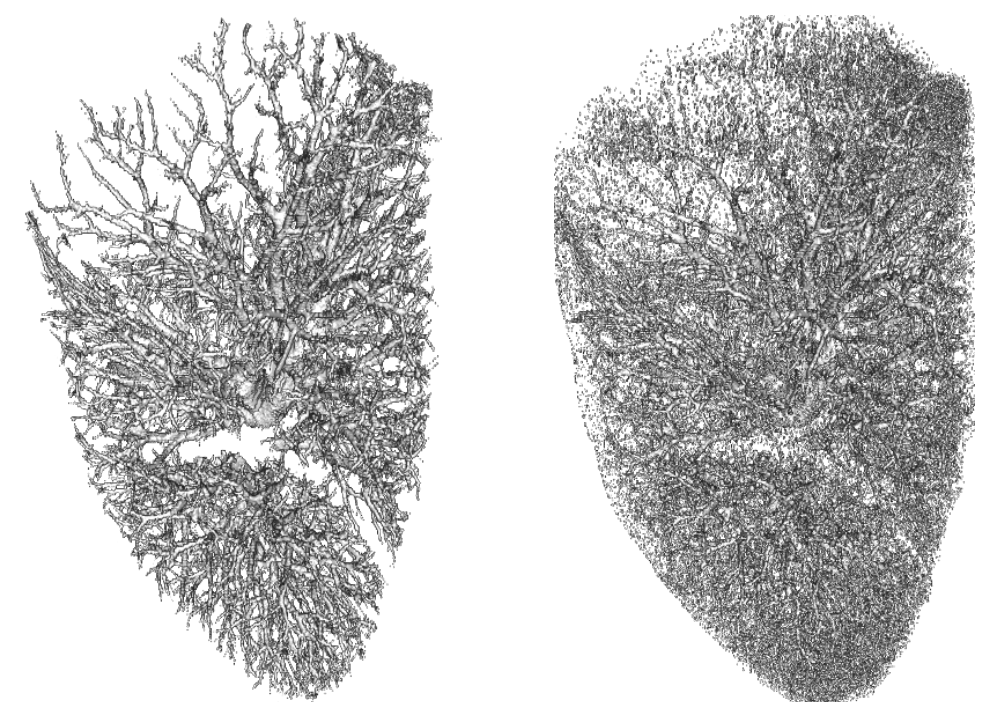

Result with connectivity constraint Without connectivity constraint

Fig. 1. Connectivity constraints allow to extract the whole branching tree of blood vessels in the lung, as shown on the left ${ }^{1}$. For comparison, a total variation regularized segmentation without connectivity constraints is shown on the right. In order to preserve the thin structures, only a very small weight of the regularizer can be chosen, therefore a lot of noise is still present in the final segmentation.

\subsection{Related Work}

Topology preserving constraints have been recently proposed for different algorithmic frameworks. For the graph cut [4] framework, Zeng et al. [17] present an extension, that allows to preserve the topology of the result with respect to an initial segmentation. Beginning on a coarse scale, their method preserves the topology of the initial segmentation during refinement. A similar approach was proposed by Han et al. [11] for the level set framework. The drawback of both methods is that they depend on the initialization and therefore only reach a local optimum.

Vicente et al. [16] introduce connectivity priors into interactive segmentation in a Markov random field framework and enforce connectivity to user given seed points. The authors show that the original problem is NP-hard and propose a greedy approximation scheme consisting of a Dijkstra algorithm where in every expansion step a graph cut needs to be solved. Their method also only reaches a local optimimum.

Chen et al. [6] propose to alternatingly solve a graph cut and modify the unary terms based on a level-set representation until predefined topological con-

\footnotetext{
${ }^{1}$ CT dataset from the Vessel Segmentation in the Lung 2012 Grand Challenge
} 
straints are fulfilled. The runtime complexity of the method prevents to use it for large scale problems.

Recently, three different methods were proposed, that aim to reach a global optimum. First, Nowozin and Lampert [12] propose to formulate the image segmentation problem with topological constraints as a linear program relaxation. However, even for small image sizes the runtime complexity of the method does not scale well and the relaxation is not tight. In contrast to the method presented in this publication, their method is not suitable for large scale problems in $3 \mathrm{D}$ segmentation.

Gulshan et al. [10] introduce geodesic star shape priors into the graph cut framework. The solution of the segmentation is restricted to the shape of a geodesic star around an input seed, while the geodesic distance depends on the image gradient. If multiple input seeds are given, the foreground segment takes the form of a geodesic forest, the union of the geodesic stars for every seed. A drawback of their method is that the boundary length regularizer is affected by the discretization of the pixel neighborhood.

In a previous work [15] we propose a global optimal segmentation method with connectivity constraints in a convex optimization framework. The combination of a total variation regularizer with a connectivity constraint allows to segment thin structures even in very noisy image data. Compared to the work of Gulshan et al. [10] our method uses a continuous segmentation framework and therefore the boundary length regularizer is not biased by discretization artifacts. The constrained optimization problem in [15] is solved by computing a solution of the dual problem. In this work, we propose an efficient projection scheme to directly compute a solution for the update of the primal variable.

\subsection{Contribution}

We propose to solve an image segmentation problem with connectivity constraints via projection onto the constraint set. We show that the constraints form a convex set and derive a projection algorithm from isotonic regression methods in statistics. We show that especially for segmentation problems with long range connections this method is by orders of magnitudes more efficient, both in iteration number and runtime, than solving the dual of the constrained optimization problem.

\section{Connectivity Constraints in Image Segmentation}

First lets review the results from [15] where image segmentation with connectivity constraints is formalized as the constrained optimization problem

$$
\begin{aligned}
& \min _{u \in B V(\Omega ;[0,1])} \int_{\Omega} f(x) u(x)+|\nabla u| d x \\
& \text { s.t. } \\
& \forall x \in \Omega, u(x)=1: \exists C_{s}^{x} \in \mathcal{G}_{s}: u\left(C_{s}^{x}(t)\right)=1 .
\end{aligned}
$$


where $I$ is an image with the domain $\Omega$, a bounded connected subset of $\mathbb{R}^{m}$, $B V(\Omega ;[0,1])$ is the space of functions with bounded variation and $f: \Omega \rightarrow \mathbb{R}$ depends on the image data. The data term $f$ is chosen in such a way that it is negative for image values which a more likely to be foreground and negative in regions which should be regarded as background, e.g. the log ratio $f(x)=\log \frac{P(I(x) \mid l(x)=0)}{P(I(x) \mid l(x)=1)}$. The discrete label assignment $l: \Omega \rightarrow\{0,1\}$, that describes if an image region belongs to the object of interest $l(x)=1$ or the image background $l(x)=0$, is relaxed by introducing the continuous indicator function $u: \Omega \rightarrow[0,1]$. The total variation regularizer $|\nabla u|$ measures the boundary length of the foreground segment. With $C_{s}^{x}$ we formalize the shortest geodesic path from a given starting point $s$, for example defined by user input, to a terminal point $x$ which is part of the geodesic shortest path tree $\mathcal{G}_{s}$.

The solution of the optimization problem should satisfy the connectivity constraint $\mathbf{C 1}$ :

For each $x \in \Omega$ that belongs to the foreground there must exist a connected shortest geodesic path from a given $s \in \Omega$ to $x$ such that all $p \in \Omega$ in the path between $x$ and $s$ belong to the foreground.

This constraint not only ensures the connection of every labeled foreground region to $s$ but also ensures that the whole foreground segment is connected.

\section{$2.1 \quad$ Geodesic Distances}

Recently, shortest geodesic distance measures have been successfully applied to image segmentation problems including medical image segmentation [3] as well as general image segmentation $[1,7]$.

In order to define the geodesic shortest path tree $\mathcal{G}_{s}$, first we have to choose an appropriate local geodesic metric. If $\lambda=0$ the labeling function $u(x)$ takes the value 1 for $f(x)<0$ and 0 for $f(x)>0$. We leave out the special case $f(x)=0$ as it does not occur in practice. For all $x_{p} \in \Omega$ that do not belong to the foreground but need to be added to the foreground to satisfy the connectivity constraint obviously $u\left(x_{p}\right)=0$ and therefore $f\left(x_{p}\right) \geq 0$. The optimal cost of the connecting path between a fixed $s$ and any $x$ in the region that should be connected on $\mathcal{G}_{s}$ is then given by

$$
\min _{C_{s}^{x}} \int_{0}^{T} f^{+}(C(t)) d t
$$

with $f^{+}=\max (0, f(x))$. Thus, we choose the non negative cost funciton $f^{+}$as metric for the construction of $\mathcal{G}_{s}$. Thus the shortest path tree can be computed using Djkstra's algorithm [8].

More complex prior models for the geodesic path are possible. In [15] we could show that a bending energy prior for the construction of the geodesic shortest path tree can improve the segmentation performance on a retinal blood vessel dataset to some extent. 


\section{Constrained Convex Optimization}

The geodesic shortest path tree forms a directed acyclic graph $\mathcal{G}_{s}=\{V, E\}$ with the set of vertices $V$ with $|V|=n$ and the set of directed edges $E \subset V \times V$ with $|E|=m$. We follow [15] and formulate the global connectivity constraint as a monotonicity constraint over each edge of this graph. To satisfy the connectivity constraint we observe that the value of the discretized value function $u_{i}$ of a node $i$ with distance to the root node $d_{i}$ should always be greater or equal than the labels of its neighbors with a larger distance $d_{j}>d_{i}$ to the root node. This implies that the directional derivative

$$
\partial_{i} u_{j}:=(d u)\left(e_{i j}\right)=(u(j)-u(i))
$$

of $u$ at vertex $i$ along the edge to vertex $j$ should always be less or equal to zero.

The image segmentation problem Eq. (1) thus can be written as the constrained optimization problem

$$
\begin{aligned}
& \min _{u_{i}[0,1]} \int_{\Omega} f(x) u(x)+\lambda|\nabla u| d x \\
& \text { s.t. } \\
& \partial_{i} u_{j} \leq 0, \forall(i, j) \in E .
\end{aligned}
$$

This image segmentation problem can be optimized using the Primal-Dual framework of $[14,5]$ which can be applied to convex optimization problems with a saddle-point structure

$$
\min _{u \in U} \max _{p \in P}\langle K u, p\rangle+G(u)-F^{*}(p)
$$

where $U$ and $P$ are finite-dimensional vector spaces, $K: U \rightarrow P$ is a continuous linear operator and $G: U \rightarrow[0,+\infty)$ and $F^{*}: P \rightarrow[0,+\infty)$ are proper, convex, lower semicontinuous functions. The update steps in [5] are computed using the prox-operator, which is defined as

$$
v=(I+\tau \partial G)^{-1}(u)=\arg \min _{v}\left\{\frac{\|u-v\|^{2}}{2 \tau}+G(v)\right\} .
$$

Using this prox-operator, the updates in the primal variable $u$ and the dual variable $p$ are computed as

$$
\begin{aligned}
& u^{k+1}=(I+\tau \partial G)^{-1}\left(u^{k}-\tau K^{*} p^{k+1}\right) \\
& p^{k+1}=\left(I+\sigma \partial F^{*}\right)^{-1}\left(p^{k}+\sigma K\left(u^{k+1}+\theta\left(u^{k+1}-u^{k}\right)\right)\right) .
\end{aligned}
$$

To formulate the image segmentation problem Eq. (3) in the Primal-Dual framework we reformulate the total variation regularizer by introducing a dual variable $p \in R^{2}[14]$ and after discretization arrive at the saddle point problem

$$
\min _{u_{i} \in[0,1]} \max _{|p| \leq 1} \lambda\langle\nabla u, p\rangle+\langle f, u\rangle+\delta_{\leq 0}\left(\nabla_{i} u\right)
$$


where $\nabla_{i} u$ is the stacked vector of the directional derivatives $\partial_{i} u_{j}$ and the connectivity constraint is included by adding its indicator function ${ }^{1}$. We identify the function $G(u)$ in Eq. (4) with $G(u)=\langle f, u\rangle+\delta_{\leq 0}\left(\nabla_{i} u\right)$.

While the constraints over the domains of $u$ and $p$ can be solved by simple projections, the optimization with respect to the connectivity constraint is more involved. In the following, we will investigate two different strategies to incorporate the connectivity constraint.

\subsection{Optimization via Fenchel Duality}

In [15] we propose to optimize the dual of the constrained optimization problem

$$
\min _{u_{i} \in[0,1]} \max _{\substack{\mid \\ \alpha \geq 0}} \lambda\langle\nabla u, p\rangle+\langle f, u\rangle+\left\langle\alpha, \nabla_{i} u\right\rangle .
$$

The connectivity constraint is ensured by introducing an additional dual variable $\alpha_{i j}$ for each edge $(i, j) \in E$. Especially for long range connections the convergence of these multipliers is very slow as we show in our experiments in section 4 .

\subsection{Projection onto the Constraint Set}

In this section we describe how the connectivity constraint can be included by directly computing the update of the primal variable subject to this constraint. Therefore we propose an efficient projection scheme to solve the constrained quadratic programming problem, which results from the definition of the proxoperator.

According to [5] the update in the primal variable $u$ is defined as

$$
\begin{aligned}
u^{k+1} & =(I+\tau \partial G)^{-1}\left(u^{k}+\tau \operatorname{div} p^{k+1}\right) \\
& =\arg \min _{v \in[0,1]}\left\{\frac{\left\|v-\left(u^{k}+\tau \operatorname{div} p^{k+1}\right)\right\|^{2}}{2 \tau}+\langle f, v\rangle+\delta_{\leq 0}\left(\nabla_{i} v\right)\right\} .
\end{aligned}
$$

By completing the square and omitting terms independent of $v$ we arrive at

$$
u^{k+1}=\arg \min _{v \in[0,1]}\left\{\left\|v-\left(u^{k}+\tau \operatorname{div} p^{k+1}-\tau f\right)\right\|^{2}+\delta_{\leq 0}\left(\nabla_{i} v\right)\right\}
$$

which is of the general form

$$
\begin{aligned}
& \arg \min _{v_{i} \in[0,1]}\|v-\tilde{u}\|^{2} \\
& \text { s.t. } \\
& v_{i} \geq v_{j}, \forall(i, j) \in E,
\end{aligned}
$$

with $\tilde{u}=\left(u^{k}+\tau \operatorname{div} p^{k+1}-\tau f\right)$.

\footnotetext{
${ }^{1}$ Note that while $\nabla_{i} u$ is defined on the graph $\mathcal{G}_{s}$, the gradient $\nabla u$ used in the total variaton regularizer is computed using standard forward operators on the image grid.
} 
Proposition 1. The feasible set $C$ determined by the constraints of the optimization problem Eq. (13) is a convex set.

Proof. Let $C_{1}$ be the feasible set determined by the inequality constraints and $C_{2}$ the constraint on the range of $v$. The feasible set of Eq. (13) then is $C=$ $C_{1} \cap C_{2}$. First we show that $C_{1}$ is convex. If for every $a, b \in C_{1}$ and $\alpha, \beta>0$ it holds that $\alpha a+\beta b \in C_{1}$ then $C_{1}$ is a convex cone. Because $a, b \in C_{1}$ it holds that

$$
a_{i} \geq a_{j}, b_{i} \geq b_{j}, \quad \forall(i, j) \in E,
$$

and because $\alpha, \beta>0$ it follows

$$
\begin{array}{ll}
\alpha a_{i} \geq \alpha a_{j}, \beta b_{i} \geq \beta b_{j}, & \forall(i, j) \in E, \\
\alpha a_{i}+\beta b_{i} \geq \alpha a_{j}+\beta b_{j}, & \forall(i, j) \in E .
\end{array}
$$

Hence the set $C_{1}$ is a convex cone. In addition to the inequality constraints we also have the constraint on the range of $v$. We call the feasible set of this constraint $C_{2}=[0,1]$. This set is convex, so $C=C_{1} \cap C_{2}$, the intersection of two convex sets, is convex.

Thus the optimization problem Eq. (13) is strictly convex subject to convex constraints. Its solution is an Euclidean projection of $\tilde{u}$ onto the set $C$ and can be solved to global optimality. Furthermore the inequality constraints describe a partial order on the values of $v$. A quadratic programming problem with this structure is known in statistics as isotonic regression [2].

\subsection{Isotonic Regression on a Tree}

In Pardalos et al. [13] the authors investigate a class of algorithms for isotonic regression where the constraints define a partial order which can be represented by a directed graph. In particular the authors propose an $O(n \log n)$ algorithm for the case when the directed graph is a directed tree with $n$ vertices. For convenience we present the algorithm IRT-BIN here as Algorithm 1.

We call the isotonic regression problem subject to partial order constraints IRT. This problem does not include the range constraints of Eq. (13). In the following, we will show that a projection of the optimal solution of IRT on the range constraint yields the optimal solution of Eq. (13).

First we follow the presentation of Pardalos et al. [13] and describe the algorithm for isotonic regression with partial order constraints, using the concept of upper sets, lower sets and level sets:

Definition 1. Let $X$ be a nonempty finite set. Let $\preceq$ be a partial order on $X$. Let $Y$ be a nonempty subset of $X$. We define the average of $Y$ as $A v(Y)=$ $\frac{1}{|Y|} \sum_{i \in Y} \tilde{u}_{i}$. We call a subset $L \subset X$ a lower set of $X$ with respect to $\preceq$ if $i \in X, j \in L$ and $i \preceq j$ implies $i \in L$. Consequently a subset $U \subset X$ is an upper set if $i \in U, j \in X$ and $i \preceq j$ implies $j \in U$. We call a subset $S \subset X$ a level 
set if there are an upper set $U$ and a lower set $L$ such that $S=L \cup U$. A block $B$ of $X$ is a nonempty level set such that for each upper set $U \subset X$ for which $U \cap B \neq \emptyset$ it holds that $A v(B) \geq A v(U \cap B)$.

Furthermore the authors of [13] introduce the concept of a block class:

Definition 2. A collection $\Delta$ of blocks of $X$ is called a block class of $X$ if

1. the blocks in $\Delta$ are pairwise disjoint and their union is the set $X$.

2. the collection $\Delta$ can be ordered by a partial-order $\preceq$ such that $A \preceq B$ for $A, B \in \Delta$ if there exist $i \in A$ and $j \in B$ such that $i \preceq j$.

Note that the collection of all singleton subsets $\{x\}$ with $x \in X$ is a block class.

The authors prove that the optimal solution of $I R T$ on a block $B$ is $v_{i}=$ $A v(B)$ for every $i \in B$. Furthermore they show that if a block class $\Delta$ has no adjacent violators, then the optimal solution of the isotonic regression is given by $v_{i}^{*}=A v(B(i))$, where $B(i)$ is the block which contains $i$, for each element $i$ of $X$.

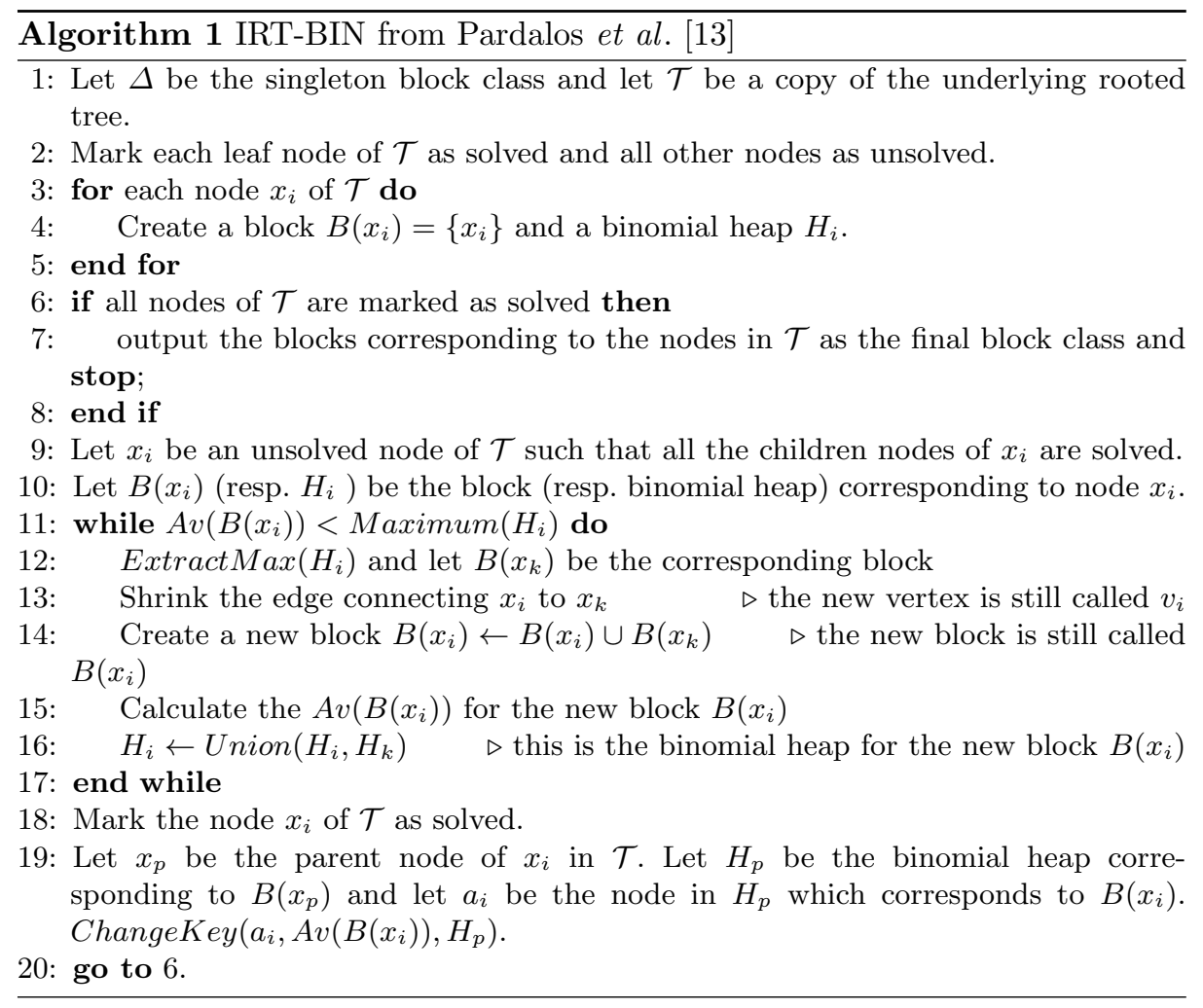

We will show with the proof of the following proposition that given a solution $v^{*}$ of IRT the optimal solution to Eq. (13) is achieved by projecting $v^{*}$ on $C_{2}$. 
Thus, we can directly project onto the constraints of the optimization problem Eq. (13) by first projecting onto the isotonicity constraint and then onto the $[0,1]$-box constraint.

Obviously, projecting first onto the $[0,1]$-box constraint and then onto the isotonicity constraint will not lead to a valid projection. When the averaging step is performed after the $[0,1]$ clipping, in case that the isotonicity constraint is violated and some values are smaller 1, only block average values well below 1 can be achieved, even when the average of the block before projection was larger than 1 .

\section{Proposition 2. Direct Projection onto the Constraint Set}

Let $B$ be a block of $X$. Let $v_{i}^{*}=A v(B)$ for every $i \in B$ be the solution of IRT. Let $\pi_{[0,1]}: \mathbb{R} \rightarrow[0,1]$ be a projection that projects negative values to 0 and values larger 1 to 1 . Then $\left\{\pi_{[0,1]}\left(v_{i}^{*}\right): i \in B\right\}$ is the optimal solution to the optimization problem (13) on $B$.

Proof. Let us assume that $B$ has $m$ elements $x_{1}, x_{2}, \ldots, x_{m}$. We look at the three cases $A v(B)>1, A v(B) \in[0,1]$ and $A v(B)<0$. Obviously these three cases are exhaustive. If $A v(B) \in[0,1]$ then the solution $v^{*}$ of IRT also fulfills the range constraint and the solution of Eq. (13) for the set $B$ is identical to the solution of IRT on $B$.

If $A v(B)>1$ we follow a similar proof as in [13] and show that the point

$$
\begin{aligned}
\left\{\pi_{[0,1]}\left(v_{i}^{*}\right): i \in B\right\} & =\left(\pi_{[0,1]}(A v(B)), \pi_{[0,1]}(A v(B)), \ldots, \pi_{[0,1]}(A v(B))\right) \in \mathbb{R}^{m} \\
& =(1,1, \ldots, 1) \in \mathbb{R}^{m}
\end{aligned}
$$

is the optimal solution to Eq. (13) by showing that the inner product of the gradient of Eq. (13) with any feasible direction $d \in \mathbb{R}^{m}$ at that point is a nonnegative number.

Let $d=\left(d_{1}, d_{2}, \ldots, d_{m}\right)$ be a feasible direction of the isotonic regression problem on $B$. Then, in order to preserve isotonicity, feasibility of the direction $d$ implies $d_{i} \leq d_{j}$ when $x_{i} \preceq x_{j}$.

Therefore there exists a permutation $\sigma=(\sigma(1), \sigma(2), \ldots, \sigma(m))$ such that

$$
d_{\sigma(1)} \geq d_{\sigma(2)} \geq \cdots \geq d_{\sigma(m)}
$$

and

$$
x_{\sigma(i)} \preceq x_{\sigma(j)} \Longrightarrow i \leq j .
$$

To prove that for $A v(B)>1$ the point in (29) is the optimal solution of the optimization problem (13) on the set $B$ it is sufficient show that

$$
\sum_{i \in B}\left(1-\tilde{u}_{\sigma(i)}\right) \times d_{\sigma(i)} \geq 0 .
$$

From Eq. (18) and from the definition of a block it follows that

$$
\frac{1}{m-k+1} \sum_{i=k}^{m} u_{\sigma(i)} \geq A v(B)>1 \text { for all } 1<k \leq m .
$$


This implies that

$$
\sum_{i=k}^{m}\left(1-u_{\sigma(i)}\right) \leq 0 \text { for all } 1<k \leq m .
$$

Equations (22) and (18) imply that for all $1<k \leq m$ that the following inequality holds

$$
\sum_{i=k}^{m}\left(1-u_{\sigma(i)}\right) \times d_{\sigma(k-1)} \geq \sum_{i=k}^{m}\left(1-u_{\sigma(i)}\right) \times d_{\sigma(k)} .
$$

Because $A v(B)>1$ the feasibility of $d$ implies that $d_{\sigma(i)} \leq 0$ for all $i \in$ $\{1, \ldots, m\}$. Combining everything together we get

$$
\begin{aligned}
& \sum_{i=1}^{m}\left(1-u_{\sigma(i)}\right) \times d_{\sigma(1)} \\
= & \sum_{i=1}^{1}\left(1-u_{\sigma(i)}\right) \times d_{\sigma(i)}+\sum_{i=2}^{m}\left(1-u_{\sigma(1)}\right) \times d_{\sigma(1)} \\
\leq & \sum_{i=1}^{1}\left(1-u_{\sigma(i)}\right) \times d_{\sigma(i)}+\sum_{i=2}^{m}\left(1-u_{\sigma(2)}\right) \times d_{\sigma(2)} \\
= & \sum_{i=1}^{2}\left(1-u_{\sigma(i)}\right) \times d_{\sigma(i)}+\sum_{i=3}^{m}\left(1-u_{\sigma(2)}\right) \times d_{\sigma(2)} \\
\leq & \sum_{i=1}^{2}\left(1-u_{\sigma(i)}\right) \times d_{\sigma(i)}+\sum_{i=3}^{m}\left(1-u_{\sigma(3)}\right) \times d_{\sigma(3)} \\
\ldots & \sum_{i=1}^{m}\left(1-u_{\sigma(i)}\right) \times d_{\sigma(i)}
\end{aligned}
$$

From $A v(B)>1$ it follows that

$$
\sum_{i=1}^{m}\left(1-u_{\sigma(i)}\right)<0 .
$$

Together with $d_{\sigma(i)} \leq 0$ for all $i \in\{1, \ldots, m\}$ it follows for Eq. (24)

$$
\sum_{i=1}^{m}\left(1-u_{\sigma(i)}\right) \times d_{\sigma(1)} \geq 0
$$

Therefore from Eq. (24) to Eq. (25) we have proven that if $A v(B)>1$

$$
\sum_{i=1}^{m}\left(1-u_{\sigma(i)}\right) \times d_{\sigma(i)} \geq 0
$$


If $A v(B)<0$ we have to show that the inner product of the gradient of Eq. (13) with any feasible direction $d=\left(d_{1}, d_{2}, \ldots, d_{m}\right) \in \mathbb{R}^{m}$ at the point

$$
\left\{\pi_{[0,1]}\left(v_{i}^{*}\right): i \in B\right\}=(0,0, \ldots, 0) \in \mathbb{R}^{m}
$$

is a positive number. This proof is equivalent to the proof for $A v(B)>1$.

\section{Experimental Results}

For comparison we performed experiments for interactive segmentation on images from [16] that also have been used in other publications, e.g. [9, 15]. As depicted in Fig. 2, the segmentations acquired with the projection method are not different from the results of the algorithm based on Fenchel duality [15].

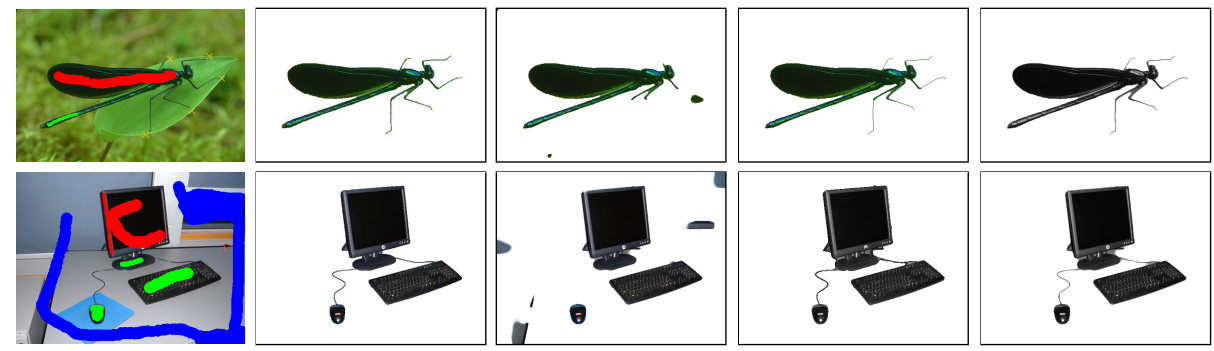

Input image Results from [16] No connectivity Fenchel Dual [15] Projection

Fig. 2. Connectivity priors for interactive segmentation. First column: Input image with user scribbles. The red scribbles are the source of the geodesic shortest path tree, green scribbles are foreground regions that should be connected and blue scribbles are background regions. Second column: Results from [16]. Third column: Segmentation without connectivity constraints. Fourth column: Segmentation with connectivity constraints by solving the dual problem [15]. Fourth column: Segmentation with connectivity constraints using the proposed projection scheme.

We provide convergence results of the two different methods on a set of synthetic test images. The set contains images of two circles that are connected by a 2 pixel wide faint path of a length of $64,128,256$ and 512 pixels. As an example, the image for the path length of 256 pixels is shown in Fig. 3.

Plots of the convergence of the two methods with respect to runtime are shown in Fig. 4. The projection method clearly outperforms the method based on Fenchel duality. The longer the connection, the higher the runtime difference of both methods. Convergence of the dual method takes from 10.12 seconds for the 64 pixel connection, over 41.11 seconds for $128,251.17$ seconds for 256 to 1639.15 seconds for the 512 pixel connection, whereas the projection method converges within less than 3 seconds for all different images. Although solving the isotonic regression problem results in a higher complexity of each iteration, by 


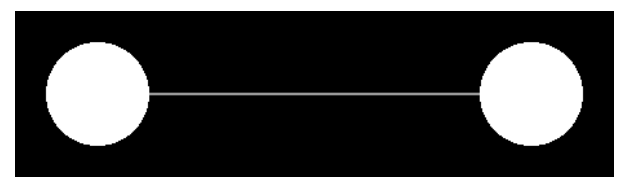

Original image

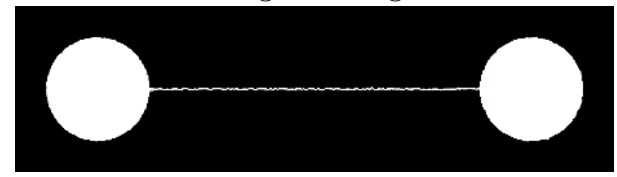

Solution of the dual problem

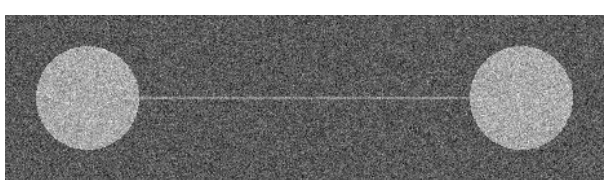

Noisy input image

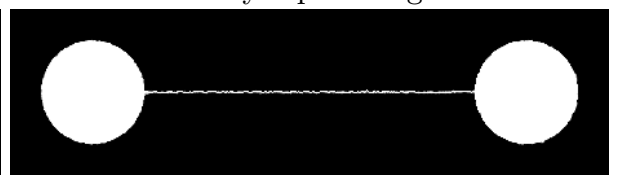

Solution using the projection method

Fig. 3. Synthetic test image. Upper row: The input image with added Gaussian noise. Lower row: Identical results of the two different methods to include the connectivity constraint.

magnitudes fewer iterations are required for the projection method to converge. The needed runtime and number of iterations until convergence for both methods are also shown in Table 1 . To measure the speed of convergence we first compute a segmentation result that is reached after a large number of iterations (10000). Then we restart the algorithm and stop when the absolute difference between the current result and the converged result is below $0.1 \%$ of the number of pixels of the image. All Experiments were performed on a a single threaded 2.27 GHZ Intel Xeon architecture.
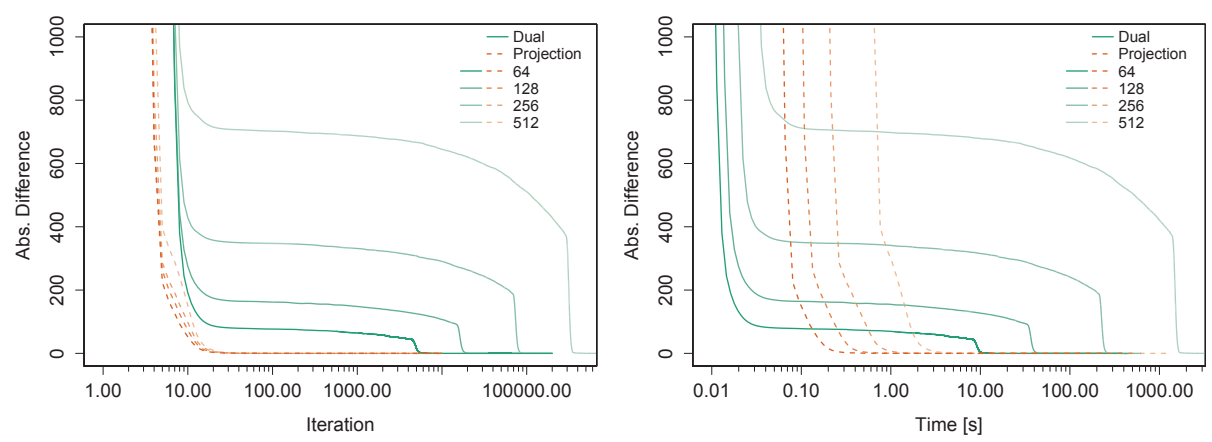

Fig. 4. Convergence of the two different methods to include the connectivity constraint on a set of test images as shown in Fig. 3. The set contains images with two circles that are connected by a 2 pixel width path of a length of $64,128,256$ and 512 pixels. Note that the plots have a logarithmic scale at the $\mathrm{x}$ axes. When using the projection method (dashed line), by order of magnitudes fewer iterations are needed than for solving the dual problem (solid). This results in a by order of magnitudes better runtime performance. 
Table 1. Comparison of runtime and number of iterations until convergence. Especially when the images contain long range connections, the projection method is by magnitudes more efficient than solving the dual problem.

\begin{tabular}{|l|rr|rr|}
\hline & \multicolumn{2}{|c|}{$\begin{array}{c}\text { Fenchel Duality } \\
\text { Iterations }\end{array}$} & Runtime & \multicolumn{2}{|c|}{ Projection } & Method \\
Image & 5396 & $10.12 \mathrm{~s}$ & $\mathbf{1 9}$ & $\mathbf{0 . 2 9} \mathbf{~ s}$ \\
Test Circle 64 & 18318 & $41.11 \mathrm{~s}$ & $\mathbf{2 0}$ & $\mathbf{0 . 5 2} \mathbf{~ s}$ \\
Test Circle 128 & 81987 & $251.17 \mathrm{~s}$ & $\mathbf{2 0}$ & $\mathbf{1 . 0 6} \mathbf{~ s}$ \\
Test Circle 256 & $3440301639.15 \mathrm{~s}$ & $\mathbf{2 0}$ & $\mathbf{2 . 8 9} \mathbf{~ s}$ \\
Test Circle 512 & 1226 & $9.13 \mathrm{~s}$ & $\mathbf{5 4}$ & $\mathbf{3 . 6 6} \mathbf{~ s}$ \\
\hline Fly & 3440 & $42.00 \mathrm{~s}$ & $\mathbf{1 0 9}$ & $\mathbf{1 3 . 4 0} \mathbf{~ s}$ \\
Desk & & & &
\end{tabular}

\section{Conclusion}

We presented a very efficient projection scheme to include connectivity constraints in a convex image segmentation framework. The method outperforms commonly used approaches that are based on Fenchel duality by orders of magnitudes. Instead of using the common approach to solve the dual problem of the constrained optimization problem we directly project onto the constraint set thus significantly fewer iterations are needed until a sufficient convergence is reached. This enables to use connectivity constraints for large segmentation problems as they arise for example in medical image segmentation of three dimensional CT angiography.

Acknowledgements We thank Michael McCoy, Michael Möller and Konstantin Pieper for fruitful discussions. This research was supported by the ERC Starting Grant "ConvexVision" and the Technische Universität München - Institute for Advanced Study, funded by the German Excellence Initiative.

\section{References}

1. Bai, X., Sapiro, G.: A geodesic framework for fast interactive image and video segmentation and matting. In: Computer Vision, 2007. ICCV 2007. IEEE 11th International Conference on. pp. 1-8. IEEE (2007)

2. Barlow, R., Brunk, H.: The isotonic regression problem and its dual. Journal of the American Statistical Association 67(337), 140-147 (1972)

3. Benmansour, F., Cohen, L.: Tubular structure segmentation based on minimal path method and anisotropic enhancement. International Journal of Computer Vision 92, 192-210 (2011), http://dx.doi.org/10.1007/s11263-010-0331-0

4. Boykov, Y., Veksler, O., Zabih, R.: Fast approximate energy minimization via graph cuts. Pattern Analysis and Machine Intelligence, IEEE Transactions on 23(11), 1222-1239 (2001)

5. Chambolle, A., Pock, T.: A first-order primal-dual algorithm for convex problems with applications to imaging. J. Math. Imaging Vis. 40(1), 120-145 (May 2011), http://dx.doi.org/10.1007/s10851-010-0251-1 
6. Chen, C., Freedman, D., Lampert, C.H.: Enforcing topological constraints in random field image segmentation. In: Proc. International Conference on Computer Vision and Pattern Recognition. pp. 2089-2096 (2011)

7. Criminisi, A., Sharp, T., Blake, A.: Geos: Geodesic image segmentation. In: Computer Vision-ECCV 2008, pp. 99-112. Springer (2008)

8. Dijkstra, E.: A note on two problems in connexion with graphs. Numerische Mathematik 1, 269-271 (1959), http://dx.doi.org/10.1007/BF01386390

9. El-Zehiry, N.Y., Grady, L.: Fast global optimization of curvature. In: CVPR. pp. 3257-3264. IEEE (2010)

10. Gulshan, V., Rother, C., Criminisi, A., Blake, A., Zisserman, A.: Geodesic star convexity for interactive image segmentation. In: Proc. International Conference on Computer Vision and Pattern Recognition. pp. 3129-3136. IEEE (2010)

11. Han, X., Xu, C., Prince, J.L.: A topology preserving level set method for geometric deformable models. Pattern Analysis and Machine Intelligence, IEEE Transactions on $25(6), 755-768$ (2003)

12. Nowozin, S., Lampert, C.H.: Global connectivity potentials for random field models. In: Computer Vision and Pattern Recognition, 2009. CVPR 2009. IEEE Conference on. pp. 818-825. IEEE (2009)

13. Pardalos, P.M., Xue, G.: Algorithms for a class of isotonic regression problems. Algorithmica 23(3), 211-222 (1999)

14. Pock, T., Cremers, D., Bischof, H., Chambolle, A.: An algorithm for minimizing the piecewise smooth mumford-shah functional. In: IEEE International Conference on Computer Vision (ICCV). Kyoto, Japan (2009)

15. Stühmer, J., Schröder, P., Cremers, D.: Tree shape priors with connectivity constraints using convex relaxation on general graphs. In: IEEE International Conference on Computer Vision (ICCV). Sydney, Australia (December 2013)

16. Vicente, S., Kolmogorov, V., Rother, C.: Graph cut based image segmentation with connectivity priors. In: Proc. International Conference on Computer Vision and Pattern Recognition (2008)

17. Zeng, Y., Samaras, D., Chen, W., Peng, Q.: Topology cuts: A novel min-cut/maxflow algorithm for topology preserving segmentation in $\mathrm{n}-\mathrm{d}$ images. Computer vision and image understanding 112(1), 81-90 (2008) 